\title{
The Phage Therapy Paradigm: Prêt-à-Porter or Sur-mesure?
}

\author{
Jean-Paul Pirnay • Daniel De Vos • Gilbert Verbeken • Maia Merabishvili • Nina Chanishvili • Mario Vaneechoutte • Martin Zizi • \\ Geert Laire $\cdot$ Rob Lavigne $\cdot$ Isabelle Huys $•$ Guy Van den Mooter • Angus Buckling $•$ Laurent Debarbieux • Flavie Pouillot • \\ Joana Azeredo • Elisabeth Kutter • Alain Dublanchet • Andrzej Górski • Revaz Adamia
}

Received: 22 July 2010 / Accepted: 27 October 2010 / Published online: 10 November 2010

(C) Springer Science+Business Media, LLC 2010

KEY WORDS antibiotic resistance · bacterial infection .

bacteriophages · drugs · phage therapy

The present opinion is the result of discussions on the future of phage therapy (personalized or large-scale uniform therapy?) during the first International Congress on Viruses of Microbes, held at the Institut Pasteur in Paris on June 21-25, 2010.

Antibiotics are becoming ineffective as important bacterial pathogens evolve to outsmart them. Yet the antibiotic pipeline is running dry with only a few new antibacterial drugs expected to make it to the market in the foreseeable future. Bacteria that are resistant to all available antibacterial drugs, so-called superbugs, are emerging worldwide. Evolutionary ecology might inform practical attempts to bring these pathogens under stronger human control (1).

\footnotetext{
J.-P. Pirnay · D. De Vos · G. Verbeken • M. Merabishvili

Laboratory for Molecular and Cellular Technology

Burn Wound Centre, Queen Astrid Military Hospital

I 120 Brussels, Belgium

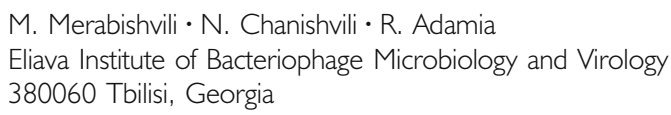

\section{Zizi}

Department of Physiology Free University Brussels

1090 Brussels, Belgium

\section{Zizi}

Section Health of the Division Well-Being (Belgian Defence Staff)

Queen Astrid Military Hospital

I 120 Brussels, Belgium
In this context, various laboratories worldwide and a handful of small pharmaceutical companies are turning to (bacterio)phages (2). Phages are natural viruses that specifically infect bacteria. They are (among) the most abundant and ubiquitous lifelike entities on Earth and coevolve with their hosts, the bacteria. Lytic phages bind to receptors on the bacterial cell surface, inject their genetic material, use the bacterium's reproductive machinery to replicate and subsequently destroy (lyse) the bacterium, irrespective of its resistance to antibiotics, releasing the newly formed phages to seek out new hosts.

In 1919, d'Herelle used phages to treat dysentery in Paris, in what was probably the first attempt to use phages therapeutically. d'Herelle eventually developed a commercial laboratory in Paris that produced phage preparations against

G. Laire

Office of the Surgeon General, Belgian Defence

I | 40 Evere, Belgium

R. Lavigne

Laboratory of Gene Technology, Katholieke Universiteit Leuven

300 I Leuven, Belgium

I. Huys

Faculty of Law, Centre for Intellectual Rights

Catholic University of Leuven

3000 Leuven, Belgium

G. Van den Mooter $(\square)$

Laboratory of Pharmacotechnology and Biopharmacy

Catholic University of Leuven

Herestraat 49

3000 Leuven, Belgium

e-mail: guy.vandenmooter@pharm.kuleuven.be 
various bacterial infections, which were marketed by what later became the large French company L'Oréal (3). In the 1930s, therapeutic phages were also marketed in the United States by major pharmaceutical companies including Eli Lilly, Squibb \& Sons (today Bristol-Meyers Squibb) and the Swan-Meyers division of Abbott Laboratories. Scientific controversies and the advent of antibiotics, however, relegated phage therapy to complete obscurity in most of the Western world. Nevertheless, phage therapy was further developed and extensively used in Eastern Europe and the former Soviet Union with activities centered at the Eliava Institute of Bacteriophage, Microbiology, and Virology (EIBMV) in Tbilisi, Georgia, several institutes in Russia, and the Hirszfeld Institute of Immunology and Experimental Therapy in Wroclaw, Poland.

Despite its long (Eastern European) history, phage therapy is not currently authorized for routine use on humans in the West. Today, it is only approved in some former Soviet republics like Russia and Georgia, where commercial phage preparations are sold in pharmacies (4). In Poland, a recent member of the European Union, phage therapy is considered an 'Experimental Treatment' covered by the Physician Practice Act (Polish Law Gazette N ${ }^{\circ} 28$ of 1997) and the

\author{
A. Buckling \\ Department of Zoology, University of Oxford \\ Oxford OXI 3PS, UK \\ L. Debarbieux \\ Department of Microbiology \\ Molecular Biology of the Gene in Extremophiles Unit \\ Institut Pasteur \\ 75724 Paris, France \\ F. Pouillot \\ Pherecydes Pharma \\ 93230 Romainville, France \\ J. Azeredo \\ Institute for Biotechnology \& Bioengineering \\ Centre of Biological Engineering \\ Universidade do Minho \\ Campus de Gualtar \\ 47 I 0-057 Braga, Portugal \\ E. Kutter \\ Phage Biology Lab, The Evergreen State College \\ Olympia, Washington 98505, USA

\section{A. Dublanchet} \\ Laboratoire de Microbiologie, Centre Hospitalier Intercommunal de \\ Villeneuve-Saint-Georges \\ 94195 Villeneuve Saint-Georges, France \\ A. Górski \\ Transplantation Institute, The Medical University of Warsaw Institute \\ of Immunology and Experimental Therapy \\ Polish Academy of Sciences Wroclaw \\ Wroclaw, Poland
}

declaration of Helsinki, where other therapeutic options do not exist (5). In France, therapeutic made-to-order phage preparations from the Institut Pasteur (Paris and Lyon) were used until the beginning of the nineties. Today, a French practitioner, Alain Dublanchet, still uses commercial phage preparations (purchased in Russia and Georgia) to treat severe infections. Despite the absence of a specific framework for phage therapy (6), a pilot clinical trial in burn wounds was approved by a leading ethical committee in Belgium (7). In the United States, a Food and Drug Administration (FDA)-approved phase I clinical trial was conducted. No safety concerns were found (7). Recently, a British phage therapy company conducted a phase I/IIa clinical trial in chronic otitis. This study was approved through the UK Medicines and Healthcare products Regulatory Agency (MHRA) and the Central Office for Research Ethics Committees (COREG) ethical review process (7).

Phages are harmless to eukaryotic (e.g. animal or plant) cells and are reported to elicit few, if any, side effects in humans. In contrast to antibiotics, they target specific bacterial species or even strains and can thus be chosen to be harmless for the non-target beneficial commensal flora (e.g. the gut flora) of the patient. This specificity also means that the right match between the phages and the targeted bacterial pathogen must be found. To improve the chance of success, off-the-shelf phage preparations should contain multiple phage strains per targeted bacterial species. This phage mixture should target the bacterial strains that are most commonly present at the intended point of use.

As with antibiotics, bacteria can evolve resistance to phages during the course of treatment (e.g. by alteration of phage receptors), to survive the phage attacks. This might result from mutations acquired during the course of treatment, but it is also likely that resistant bacteria are already present in the target population before phage treatment. Indeed, it is not in phages' best interest to kill all the host bacteria in the infection site, but they can be expected to (bio)control the bacterial pathogens and significantly reduce their numbers and thus give the patient's immune system and/or antibiotics the chance to eliminate the remaining bacteria. Moreover, in vitro, natural selection drives the rapid emergence of new phages that can destroy bacteria that have become resistant (9), and this may also be important in clinical contexts.

Ninety years of phage therapy have shown that after a while phage preparations become less effective and need to be updated. The ineffective phages can either be "trained," a term used in the EIBMV to indicate the selection of phage mutants more active against the phage-resistant bacteria, or replaced by new active phages. New phages are generally selected from the environment (e.g. sewage water), but in some cases they can be isolated from clinical 
samples containing the problematic bacterium. In phage therapy centers in Georgia and Poland, banks containing many different phages are kept and regularly updated. Sometimes custom phage preparations are developed for a patient's infection (autophage), a procedure that usually takes a few days to weeks.

This sur-mesure approach is not compatible with the current licensing processes. Recently, the European Med- icines Agency (EMA) placed phages under the Medicinal Product Regulation and more specifically under the category of biologicals. Also, in the US, the amount of research and testing required by the FDA is seriously hampering the resurgence of phage therapy. Regulators impose many years of research and clinical trials, which cost millions of euros, to entrepreneurs to develop and distribute phage preparations (Fig. 1).

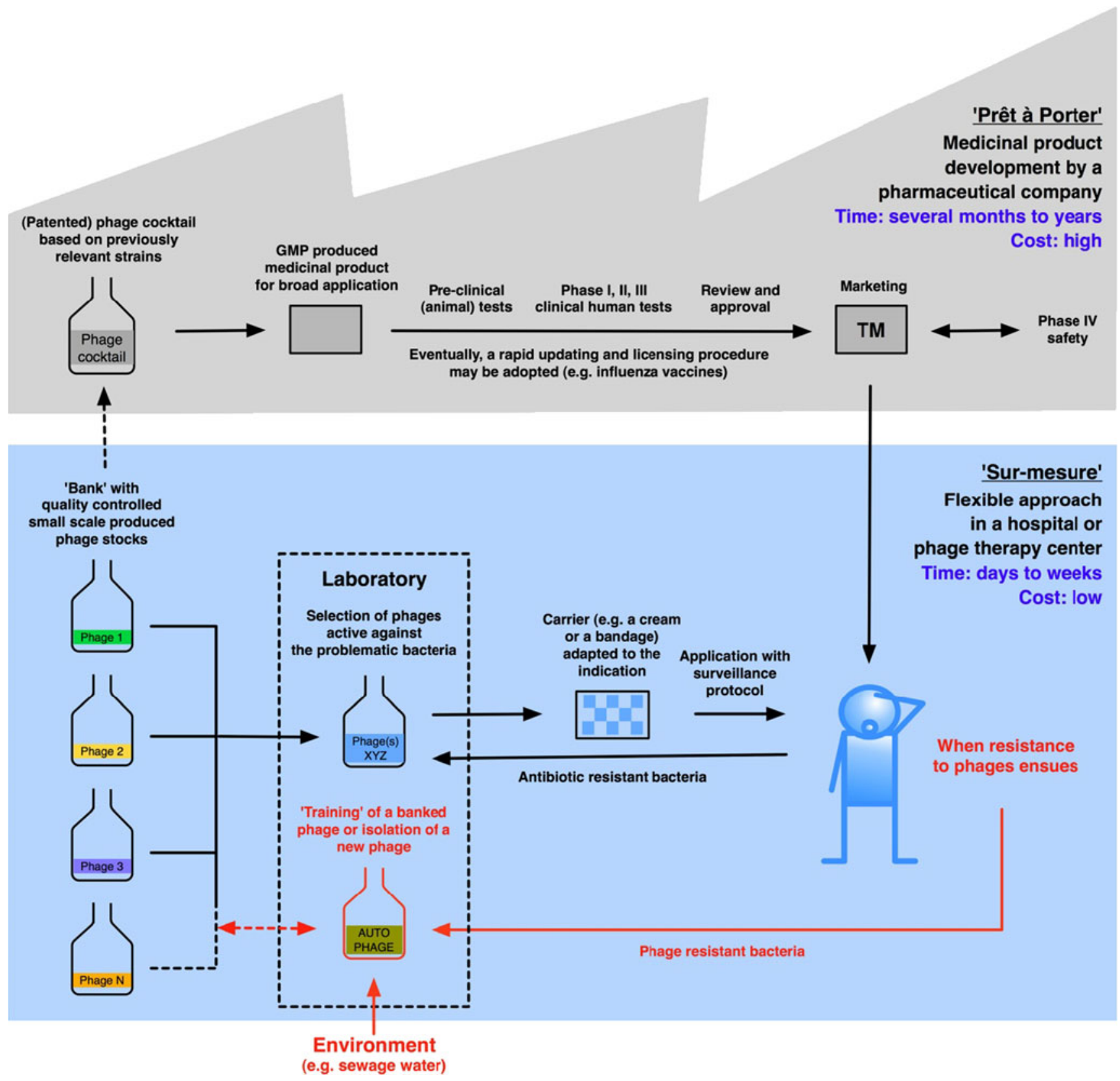

Fig. I Phage therapy concepts: prêt-à-porter or sur-mesure? Both approaches should be possible and could even be complementary. The specificity of phages, resistance and IP issues may thus hamstring pharmaceutical companies in the worldwide marketing of generic phage preparations. The long and expensive regulatory pathways, on the other hand, form insurmountable obstacles for bonafide or non-profit phage therapy centers or hospitals, which opt for a sur-mesure concept, and for institutions that would like to use inexpensive phages for commercially unattractive applications, in emerging countries, for example. Of course, this sur-mesure approach should also adhere to certain standards of behavior, safety and quality control (8). These standards could be defined in a new and specific section for phage therapy under the Advanced Therapy Medicinal Product Regulation (6). 
Notwithstanding these regulatory hurdles and the empirical evidence suggesting that stable and widely distributed phage preparations (prêt-à-porter) will only be of (time-)limited use, a few companies have picked up the gauntlet and are moving along the elaborate and expensive licensing pathways. If nothing else, these efforts will put phage therapy back on the map in the Western world, and, once commonly accepted, EMA and FDA might revise their rules the way they did for influenza vaccines, which also require a rapid updating and licensing procedure (10). However, are pharmaceutical companies willing to commit to rapidly and regularly adapting their phage preparations to very specific or newly emerging demands? Take, for example, a hospital unit confronted with an MDR bacterial strain that causes untreatable infections in only one or two patients. Phage therapy is probably best-but not exclusively — served by small-scale productions and distributions of locally adapted or personalized phage preparations (cottage industry) (Fig. 1).

To avoid the drug licensing pathway, some US-based phage companies decided to first develop phage products for the decontamination of food, plants, fields and livestock (2). They hope to create revenue to fund research into human therapeutics and to familiarize the authorities and the general public with phages. Phages for decontaminating food plants, ready-to-eat meat, poultry products, cheese and live animals that will be slaughtered for human consumption were approved by the FDA and are now in use. Very little is known, however, about the impact of such massive and widespread applications of phages on natural microbial communities.

The lack of strong intellectual property (IP) protection is another discouraging factor for pharmaceutical companies. The principle of phage therapy has been common knowledge since the 1920s, and many aspects might thus be unpatentable. In addition, there are indications that in the future, phages, which are natural entities composed of genetic material and proteins, will only be patentable if they have been engineered into something distinctly different in character (11). Engineered phages could be patented, but, considering the current concerns about potential risks for public health and the environment which may arise from genetic engineering in genetically modified organisms (GMOs), they are not likely to be given licensing approval in the near future. Phage-derived products (e.g. cell wall-degrading enzymes such as endolysins) can and probably will be licensed and marketed within a few years. They may also select resistance, but presumably at a lower rate than antibiotics. Of course, these phage products lack the capacity of self-replication and adaptation in the infectious site.

Phage therapy has great potential in some (niche) clinical contexts, but as with antibiotic treatment, there are likely to be important evolutionary consequences (12) if it is implemented widely and without sufficient oversight. Some aspects of phagebacterium evolution ecology (e.g. emergence of resistance) should first be analyzed in the light of future phage therapy. Real-time experimental evolution studies could help determine these evolutionary consequences and generate the analytical knowledge in support of the empirical knowledge and clinical experience that was accumulated in the Eastern world. More importantly, they will hopefully enable the creation of a rational phage therapy concept (Fig. 1), thus avoiding the historical mistakes that occurred in the course of antibiotic therapy development and which lead to the current massive and widespread occurrence of antibiotic resistance in the patient population as well as in the natural environment.

\section{REFERENCES}

1. Williams PD. Darwinian interventions: taming pathogens through evolutionary ecology. Trends Parasitol. 2010;26:83-92.

2. Thiel K. Old dogma, new tricks-21st Century phage therapy. Nat Biotechnol. 2004;22:31-6.

3. Sulakvelidze A, Alavidze Z, Morris Jr JG. Bacteriophage therapy. Antimicrob Agents Chemother. 2001;45:649-59.

4. Chanishvili N, Sharp R. Eliava Institute of Bacteriophage, Microbiology and Virology, Tbilisi, Georgia. A literature review of the practical application of bacteriophage research. Tbilisi: Eliava Foundation; 2009.

5. Górski A, Miedzybrodzki R, Borysowski J, Weber-Dabrowska B, Lobocka M, Fortuna W, et al. Bacteriophage therapy for the treatment of infections. Curr Opin Investig Drugs. 2009;10:766-74.

6. Verbeken G, De Vos D, Vaneechoutte M, Merabishvili M, Zizi M, Pirnay J-P. European regulatory conundrum of phage therapy. Future Microbiol. 2007;2:485-91.

7. Kutter E, De Vos D, Gvasalia G, Alavidze Z, Gogokhia L, Kuhl $\mathrm{S}$, et al. Phage therapy in clinical practice: treatment of human infections. Curr Pharm Biotechnol. 2010;11:69-86.

8. Merabishvili M, Pirnay J-P, Verbeken G, Chanishvili N, Tediashvili M, Lashkhi N, et al. Quality-controlled small-scale production of a well-defined bacteriophage cocktail for use in human clinical trials. PLoS ONE. 2009;4:e4944.

9. Buckling A, Rainey PB. Antagonistic coevolution between a bacterium and a bacteriophage. Proc Biol Sci. 2002;269:9316.

10. Wood JM, Levandowski RA. The influenza vaccine licensing process. Vaccine. 2003;21:1786-8.

11. Akst J. Key cancer patents killed. http://www.the-scientist.com/ blog/display/57265/ (accessed 19/10/10), part of The Scientist. http://www.the-scientist.com/ (accessed on 19/10/10).

12. Levin BR, Bull JJ. Population and evolutionary dynamics of phage therapy. Nat Rev Microbiol. 2004;2:166-73. 